Anales de Geografía de la Universidad Complutense ISSN: 0211-9803

http://dx.doi.org/10.5209/AGUC.72985

\title{
Las exigencias ambientales de la última reforma de la Política Agraria Común (2014-2020) de la Unión Europea: conflictos, desequilibrios e incongruencias
}

\author{
José Antonio Segrelles Serrano ${ }^{1}$ \\ Recibido: 11 de junio del 2020 / Enviado a evaluar: 17 de junio del 2020 / Aceptado: 15 de diciembre del 2020
}

Resumen. Durante el desarrollo de la aplicación de la Política Agraria Común (PAC) de la Unión Europea (UE) y sus sucesivas reformas se percibe un progresivo cambio de paradigma que va desde los enfoques netamente productivos a otros presididos por una preocupación ambiental, sostenible y multifuncional de los espacios rurales. Es en la última reforma de la PAC, que estará en vigor en el periodo 2014-2020, donde se apuesta de manera clara por una actividad agropecuaria respetuosa con el ambiente y el clima, si bien surgen conflictos y desequilibrios entre la búsqueda de una agricultura competitiva y comercial y la defensa de los bienes públicos asociados a ella. El objetivo principal estriba en poner de relieve las contradicciones, conflictos, incongruencias y desequilibrios que alberga la última reforma de la PAC por lo que respecta a sus nuevas exigencias ambientales. La PAC reformada no ha tenido en cuenta las particularidades agropecuarias y forestales de las diferentes zonas agropecuarias de la UE, motivo por el que se ocasionan antagonismos, injusticias y desequilibrios territoriales, agrarios y socioeconómicos entre los países miembros. Los países más afectados son los mediterráneos, especialmente España.

Palabras clave: Política Agraria Común; Unión Europea; última reforma; periodo 2014-2020; exigencias ambientales.

[en] A consideration of the environmental requirements of the latest reform of the European Union Common Agricultural Policy (2014-2020): conflicts, imablances and incosistencies

\begin{abstract}
When implementing the Common Agricultural Policy (CAP) of the European Union (EU) and its successive reforms a progressive change in paradigm was perceived ranging from markedly productive approaches to others with a concern for environmental, sustainable and multifunctional concern for rural areas. The CAP's most recent reform, which will be in force from 2014-2020, clearly aims to ensure farming activity which respects the environment and the climate, although there are some conflicts and imbalances between seeking to achieve a competitive and commercial agriculture and the defence of the public assets associated with those practices. The main objective is to highlight the
\end{abstract}

1 Departamento de Geografía Humana - Universidad de Alicante. España.

E-mail: ja.segrelles@ua.es 
contradictions, conflicts, inconsistencies and imbalances found in the latest CAP reform relating to its new environmental demands. The reformed CAP has failed to consider the agricultural and forestry specificities proper to the EU's different farming areas, which explains antagonisms, injustices and territorial, agrarian and socio-economic imbalances among Member States. The most affected countries are in the Mediterranean, especially Spain.

Keywords: Common Agricultural Policy; European Union; latest reform 2014-2020; environmental requirements.

\section{[fr] Les exigences environnmentales de la derniere reforme de la Politique Agricole Commune (2014-2020) de L'Union Europeenne: conflits, desequilibres et incoherences}

Résumé. Pendant le développement de la mise en pratique de la Politique Agricole Commune (PAC) de l'Union Européenne (UE) et ses réformes successives se perçoit un changement progressif de paradigme qui va depuis des approches nettement productives à d'autres animées par une préoccupation environnementale, soutenable et multifonctionnelle des espaces ruraux. C'est dans la dernière réforme de la PAC, en vigueur sur la période 2014-2020, qu'elle se positionne de façon claire pour une activité agropastorale respectueuse de l'environnement et du climat, bien que surgissent des conflits et des déséquilibres entre la recherche d'une agriculture compétitive et commerciale et la défense des biens publics qui lui sont associés. L'objectif principal repose sur la mise en exergue des contradictions, conflits, incohérences et déséquilibres que porte la dernière réforme de la PAC quant à ses nouvelles exigences environnementales. La PAC réformée n'a pas pris en compte les particularités agropastorales et forestières des différentes zones agricoles de l'UE, raison pour laquelle s'opèrent antagonismes, injustices et déséquilibres territoriaux, agraires et socio-économiques entre les pays membres. Les pays méditerranéens sont les plus affectés, en particulier l'Espagne.

Mots clés: Politique Agricole Commune; Union Européenne; dernière réforme; période 2014-2020; exigences environnementales.

Cómo citar. Segrelles Serrano, J.A. (2020): Las exigencias ambientales de la última reforma de la Política Agraria Común (2014-2020) de la Unión Europea: conflictos, desequilibrios e incongruencias. Anales de Geografía de la Universidad Complutense, 40(2), 541-559.

Sumario. 1. Introducción. 2. Una breve aproximación a las primeras preocupaciones ambientales de la PAC y sus reformas. 3. El ambiente y la última reforma de la PAC: el "Pago Verde" y sus condiciones. 4. Amenazas ambientales y sociales de la Nueva PAC para el medio rural español. 5. Conclusiones. 6. Bibliografía. 7. Recursos electrónicos.

\section{Introducción}

La frase de la periodista, escritora e investigadora canadiense N. Klein (2015) con la que se inicia este artículo es suficientemente elocuente de lo difícil que resulta conciliar el respeto al ambiente y la protección de los recursos naturales con el deseo compulsivo de conseguir la máxima productividad en cualquier actividad económica que se realice y con la necesidad de alcanzar un crecimiento constante, exigencia esta última más dogmática que otra cosa para el modelo socioeconómico imperante.

Dicha conciliación entre la economía y la naturaleza resulta más complicada cuando se trata de la agricultura y la ganadería, actividades que por definición se desarrollan en un medio natural y que no ofrecen las condiciones de un verdadero mercado. 
Estas cuestiones llaman poderosamente la atención cuando se trata de analizar la evolución de la Política Agraria Común (PAC) de la Unión Europea (UE) durante las últimas décadas $\mathrm{y}$, ante todo, el alcance ambiental de la nueva reforma de dicha política común de 2013 planteada para el periodo 2014-2020. De este modo, la PAC ha pasado de unos planteamientos de productividad a toda costa a convertirse en una política impulsora de una visión sostenible, multifuncional, interdependiente y ambiental del medio rural. Se puede decir entonces que la PAC ha sido el brazo ejecutor de la UE, implantando y desarrollando los cambios paradigmáticos que justifican sus acciones a la hora de apoyar a las actividades agrarias y a los territorios rurales para darles una orientación u otra.

La UE siempre ha sabido argumentar con convicción los cambios y nuevos horizontes de sus políticas agrorrurales, tanto por medio de sus publicaciones y documentos como a través de los medios de comunicación de masas y de los políticos comunitarios y nacionales. Por eso resulta tan complicado escuchar algunas voces críticas con las causas y consecuencias de dichas políticas agrarias y rurales. De los políticos no se puede esperar demasiado y no cabe decir sobre ello mucho más, pero lo cierto es que tampoco abundan los estudiosos y científicos sociales que vayan más allá de las declaraciones oficiales y cuestionen las decisiones políticas, y por ende socioeconómicas, que afectan al campo europeo. La PAC suele ser analizada con bastante condescendencia y haciendo hincapié en sus aspectos positivos ${ }^{2}$. Eso sí, una vez pasado el tiempo no hay mayor problema en reconocer sus errores, como sucede en el caso de la contaminación y las notables agresiones ambientales derivadas del fomento de una productividad a ultranza en los primeros momentos, tras su creación como política común, tal vez porque en aquellos años era necesario actuar de esta manera. En cualquier caso, los más críticos con la PAC y sus consecuencias ambientales suelen ser siempre algunas organizaciones no gubernamentales especialmente concienciadas, como por ejemplo Oxfam International ${ }^{3}$ o Ecologistas en Acción ${ }^{4}$.

${ }^{2} \mathrm{Al}$ respecto puede consultarse el amplio trabajo colectivo coordinado por Isabel Bardají (2014) o la aportación, también colectiva, auspiciada por la Fundación de Estudios RuralesUnión de Pequeños Agricultores (2013), con la colaboración del Ministerio de Agricultura, Alimentación y Medio Ambiente de España.

${ }^{3}$ El documento de debate La necesidad de ser coherentes. Reforma de la PAC y países en desarrollo (junio de 2002, 16 p.) ha sido elaborado por G. Fanjul, con la contribución de Celine Charveriat, y forma parte de la campaña de Oxfam International "Comercio con justicia".

Disponible

$<$ http://fongdcam.org/manuales/comerciojusto/datos/docs/ARTICULOS\%20Y\%20DOCUME NTOS $\% 20$ DE $\% 20$ REFERENCIA $\% 20 \mathrm{def} / \mathrm{CONCEPTO} \% 20 \mathrm{CI} / \mathrm{Como} \% 20$ funciona $\% 20 \mathrm{el} \% 20$ sistema/12-coherencia\%20PAC.pdf>. [Consulta: 27 de agosto de 2017].

4 Artículo de J. Ferrer (2005) en la Revista El Ecologista, no 46. Disponible en: $<$ http://www.ecologistasenaccion.es/article8133.html $>$. [Consulta: 27 de agosto de 2017]. 
En la actualidad, ante la aplicación de la nueva reforma de la PAC para el periodo 2014-2020, la Comisión Europea (2014: 11) sostiene que ya no tiene sentido conseguir grandes producciones agropecuarias para alimentar a la población europea. De ahí la consolidación del discurso ambiental y la orientación de la PAC para ayudar a los agricultores a conseguir las siguientes metas: empleo de técnicas agropecuarias ecológicas, protección del ambiente, preservación de los ecosistemas, reducción de las emisiones de contaminantes a la atmósfera, producción de alimentos sanos e inocuos, decantación hacia los productos genuinos del lugar de cultivo o cría, fomento del uso sostenible de montes y bosques, entre otras.

Sin embargo, en los discursos oficiales autocomplacientes y optimistas existen realidades que no suelen comentarse, ya que la PAC encierra muchas contradicciones y actúa en numerosas ocasiones en sentido contrario al que publicita. A veces parece que no le interesa solucionar los antagonismos que generan sus decisiones en el medio rural y entre los distintos agentes que participan en el complejo mundo de la producción agropecuaria. Incluso se percibe un cierto cinismo al intentar solucionar de manera marginal y con subvenciones públicas los daños que sobre el territorio rural, el ambiente, los ecosistemas y la agricultura familiar ocasionan el mercado neoliberal y los fuertes procesos de concentración productiva que tienen lugar, con su impulso, en las regiones más desarrolladas de Europa, aspectos éstos que constituyen la hipótesis de partida de esta investigación. Baste observar cómo reparte la PAC las subvenciones y los criterios que sigue para concederlas para comprender que la propia UE contribuye a la creación de estos antagonismos y a la perpetuación del estado de cosas tradicional pese al nuevo discurso ambiental (Segrelles, 2017). La denuncia de estos problemas también son moneda corriente entre algunos investigadores europeos, y además resulta curiosa la frase que utilizan en inglés $\mathrm{C}$. Rutz, J. Dwyer y J. Schramek (2013: 1) en la primera parte del título de su artículo para indicar esa política lampedusiana de que todo debe cambiar para que todo siga igual: "More new wine in the same old bottles?".

Detrás de los optimistas discursos oficiales, que abogan por una agricultura respetuosa con el ambiente, se esconden contradicciones flagrantes en unas acciones que van precisamente en sentido contrario al que se supone que se desea impulsar. Por ejemplo, no se puede abogar por la preservación y protección ambiental y luego subvencionar con generosidad a las explotaciones más industrializadas, que son las que más contaminan, o permanecer impasible ante la desaparición de millones de explotaciones familiares, es decir, las más pequeñas, las que crean empleo, las que generan riqueza en la economía rural y las que aprovechan los recursos locales conservando la agrobiodiversidad. De ahí que algunos autores (Delgado, 2004; Roca y Ricart, 2014) hagan hincapié en el dilema que para las políticas agrorrurales europeas más recientes supone la competitividad de la agricultura o la protección de los bienes públicos asociados a ella, es decir, todo aquello que no valora ni regula el mercado, como sucede con el mantenimiento del paisaje, la integridad del territorio y 
los ecosistemas, el fomento de la biodiversidad, la protección de los suelos, la atmósfera y las aguas superficiales y subterráneas, la lucha contra la erosión, la defensa de la flora y la fauna, entre otros ${ }^{5}$.

Las ideas que aquí se exponen se insertan en una línea de pensamiento que se apoya en el razonamiento de que la economía de mercado tiene una capacidad sin parangón para generar riqueza, pero también para concentrarla en unas clases sociales, en unas actividades y en unos territorios en detrimento de otros. Es en el desorden y el desequilibrio aparentes donde el capital se siente cómodo y se mueve a sus anchas para obtener beneficios y luego acumularse y reproducirse. La nueva reforma de la PAC (2014-2020) viene a consolidar este estado de cosas, ya planteado en sus anteriores reformas (1992, 1996, 1997, 2003 y 2008), y a establecer antagonismos más acusados entre el norte y el sur de la UE y entre la actividad agraria familiar y la agricultura comercial y capitalizada. Y todo esto bajo el discurso asumible por la sociedad del desacoplamiento de las ayudas, la sostenibilidad, la multifuncionalidad y el desarrollo rurales, la protección del ambiente y las buenas prácticas agrícolas en las explotaciones.

\section{Una breve aproximación a las primeras preocupaciones ambientales de la PAC y sus reformas}

En sus inicios y con el objeto de aumentar las producciones, la UE por medio de la PAC promovió la creciente mecanización del campo y el uso de pesticidas y fertilizantes químicos, introdujo regadíos en secanos históricos (maíz, viñedo, olivar), redujo la ganadería extensiva y fomentó la industrializada, concentró la producción en determinadas regiones para orientarla a los mercados globales y dejó en el abandono a otras zonas menos competitivas. El resultado más inmediato fue una progresiva contaminación del agua y los suelos, la contribución al calentamiento global, la erosión de la tierra, la destrucción de muchos ecosistemas, la desaparición de recursos genéticos autóctonos, la cuestionable calidad de los alimentos y la destrucción de millones de pequeñas y medianas explotaciones, hecho especialmente acentuado en los países meridionales de la UE, como es el caso de España.

Desde las primeras reformas en 1992 y después en 1996 con la Declaración de Cork y en 1997 con la Agenda 2000 (Comisión Europea, 1997), la PAC ha ido profundizando y ampliando sus exigencias ambientales a las explotaciones agropecuarias de la UE. Unos factores decisivos para que Bruselas se propusiera reformar la PAC y darle una orientación más "verde" fueron sin lugar a dudas los insostenibles gastos presupuestarios de esta estrategia europea de intervención pública y la excesiva producción de excedentes en el campo, así como las protestas constantes

\footnotetext{
${ }^{5}$ Precisamente, el prólogo del libro de M. M. Delgado (2004), redactado por E. Ramos, lleva por título "La política rural europea entre la competitividad y los bienes públicos".
} 
de los principales países exportadores de materias primas agroalimentarias, que siempre han acusado a la UE de competencia desleal (dumping) en los mercados internacionales al subvencionar sus producciones agrícolas y ganaderas.

La PAC fue dejando de lado las clásicas políticas sectoriales y productivas para centrarse en los objetivos ambientales y en una visión global y multifuncional del medio rural. El sector agropecuario pierde protagonismo en un espacio rural cada más heterogéneo y complejo.

La PAC intenta, por medio de sus nuevas directrices ambientales y multifuncionales, que el medio rural comunitario y su agricultura se adapten a las estrictas exigencias de la Organización Mundial del Comercio (OMC) en cuanto a la limitación o supresión de todas aquellas ayudas que puedan distorsionar el comercio agroalimentario internacional. Tanto el ambiente como los recursos naturales se presentan una vez más como coartada para lograr otros fines de tipo económico, financiero, geopolítico y mercantil.

En cualquier caso, el hito fundamental en el devenir de la PAC y en su viraje hacia la ejecución de políticas ambientales tuvo lugar con la reforma del año 2003 y con la revisión, o "chequeo", de la misma que se produjo en 2008, momento previo de la reforma de 2013, que es la que se encuentra en vigor en la actualidad.

Tras varias reformas de la PAC, cuyo norte principal era corregir las distorsiones del mercado y adaptarse a las directrices de la OMC, a partir de 2003 el $90 \%$ de sus fondos se desvincularon o desacoplaron de la producción para ligarse sólo a la cantidad de tierra cultivada. Otro instrumento nuevo de la reforma de 2003 fue el de la llamada eco-condicionalidad agraria, es decir, la concesión de las subvenciones públicas estaría vinculada a los programas de desarrollo rural integral y multifuncional, la pluriactividad campesina, la sostenibilidad de la agricultura y las buenas prácticas agropecuarias, esto es, el respeto ambiental, el fomento de los sistemas agrícolas y ganaderos extensivos, la agricultura ecológica y la permacultura, la producción de alimentos sanos e inocuos para la salud de los consumidores, la protección del paisaje y la biodiversidad, la salvaguarda de los recursos naturales, el bienestar animal, entre otros.

Por su parte, los cambios acontecidos en 2008, tal como indica M. Fischer-Boel (2008), no representaron una nueva PAC, pues en realidad se trataba de un impulso para racionalizar y modernizar esta política agraria. Ante todo, se buscaba la eliminación de todos los impedimentos que suponían un importante obstáculo para que los productores europeos pudieran interpretar con acierto las señales de los mercados y afrontar el aumento creciente de la demanda alimentaria.

Esto demuestra de manera elocuente que Bruselas quiere, por medio de la PAC, hacer compatible sus exigencias ambientales con la necesidad de que sus empresas agroalimentarias, la mayoría de carácter transnacional, sean competitivas a nivel global, y con el interés apenas disimulado de aumentar las exportaciones de sus productos, sin tener en cuenta que el respeto ambiental, por un lado, y el impulso exportador y el afán competitivo, por otro, encierran conceptos claramente antagónicos. 
En ningún momento, ni la reforma de 2003 ni la de 2008 cuestionaron, por ejemplo, el modelo ganadero industrializado. No se actuó para desincentivar la producción intensiva de carne, leche y huevos pese a toda la propaganda ambiental y sostenible. La UE sigue apoyando este sistema productivo intensivo con sus políticas porque la inexistencia de aranceles para las importaciones de soja garantiza una oferta suficiente a bajo precio para las grandes estructuras empresariales que controlan la ganadería industrializada europea y su alimentación a base de piensos compuestos.

$\mathrm{Si}$ las cuestiones ambientales preocuparan verdaderamente a la UE, la PAC no apoyaría con fondos públicos a la ganadería industrial, que es cualquier cosa menos sostenible y respetuosa con el medio, e impulsaría de manera decidida los sistemas pecuarios extensivos más beneficiosos para el ambiente, el clima ${ }^{6}$, la salud de los consumidores y las comunidades rurales dinámicas y vivas. Como se dice en un reciente informe de la organización no gubernamental Greenpeace (2019), los subsidios de la PAC han promovido los sistemas de producción agropecuaria intensivos. Incluso más de la mitad del presupuesto total de la PAC está vinculado al sector ganadero industrial.

\section{El ambiente y la última reforma de la PAC: el "Pago Verde" y sus condiciones}

Impelida por circunstancias de tipo socioeconómico y por el contexto económico y comercial mundial, la PAC se ha transformado en su última reforma para el periodo 2014-202 en una política agraria cada vez más "verde", de forma que se establece el pago para aquellas prácticas agropecuarias que no sean perjudiciales para el ambiente ni para el clima. Este pago también es conocido como "pago verde" (o greening, en inglés) y permite conceder un pago anual por cada hectárea admisible vinculada a un derecho de pago básico, siempre que se respeten determinadas prácticas ambientales y dependiendo de la estructura de la explotación, según se indica en una nota técnica $\left(\mathrm{N}^{\mathrm{o}}\right.$ 03) difundida por el Ministerio de Agricultura, Alimentación y Medio Ambiente (2015 b) de España.

En la mencionada nota técnica se señala que el importe del "pago verde" es un porcentaje del valor total de los derechos de pago básico que active el agricultor de manera anual. Dicho porcentaje, que lo normal es que sea superior al $50 \%$, se determinará cada año. Este pago será sufragado por las arcas públicas comunitarias a partir del 30\% del límite presupuestario correspondiente a cada país miembro. En el año 2015 este presupuesto ascendió, en el caso de España, a 1.453 millones de euros,

${ }^{6}$ La ganadería, sobre todo la intensiva, tiene un impacto muy serio sobre el ambiente, el clima y la salud de los consumidores. Si se redujera a la mitad el consumo de carne, huevos y productos lácteos en la UE, sus emisiones de gases de efecto invernadero a la atmósfera (metano fundamentalmente) podría rebajarse entre un $25 \%$ y un $40 \%$, según $\mathrm{H}$. Westhoek et al (2014). 
cifra que se prevé que aumente ligeramente hasta alcanzar 1.468 millones de euros en 2019.

Otra nota técnica ( $\mathrm{N}^{\mathrm{o}}$ 07) del Ministerio de Agricultura, Alimentación y Medio Ambiente (2015 a) de España, relacionada con la nueva orientación ambiental de la última reforma de la PAC, explica la condicionalidad, es decir, las obligaciones que se deben observar con el fin de que no mengüe el importe de las subvenciones europeas al sector agrario y que se relacionan con la naturaleza, las buenas condiciones de la tierra, la salud pública, la sanidad vegetal y el bienestar del ganado. Esta condicionalidad afecta a los agricultores y ganaderos que reciben pagos directos, ayudas a la reestructuración y reconversión del viñedo, ayudas a la cosecha en verde o a algunos de los siguientes elementos relacionados con el desarrollo rural: reforestación y creación de superficies forestales, implantación de sistemas agroforestales, agroambiente y clima, agricultura ecológica, ayudas a las zonas con limitaciones naturales $\mathrm{u}$ otras limitaciones concretas, pagos al amparo de la Red Natura 2000 y de la Directiva Marco del Agua, y servicios silvoambientales, climáticos y de conservación de los bosques.

La totalidad de los beneficiarios con derecho a pago en virtud del régimen de pago básico deben respetar tres prácticas ambientales en todas las hectáreas admisibles que tenga la explotación. Estas prácticas son las siguientes: diversificación de cultivos, mantenimiento de los pastos permanentes existentes y presencia de superficies de interés ecológico en las unidades de producción. Por su parte, tendrán derecho a un pago automático los agricultores que se dedican a la agricultura ecológica, los que están acogidos al régimen de pequeños agricultores y que, por lo tanto, no cobran más de 1.250 euros de pagos directos, y los que disponen de cultivos permanentes: cítricos, olivar, frutales, viñedo y, en general, aquellos que permanecen en el terreno durante cinco o más años y que no entran en la rotación de cultivos de la explotación.

La diversificación de cultivos consiste en sembrar varios cultivos diferentes ${ }^{7}$ en la superficie labrada de la explotación, es decir, la tierra ocupada por cultivos herbáceos de secano o de regadío, que de modo habitual se cosechan anualmente, o dejada en barbecho. Esta diversificación implica que si la tierra de cultivo cubre entre $10^{8}$ y 30

\footnotetext{
${ }^{7}$ Se consideran cultivos diferentes los que pertenecen a distintos géneros botánicos.

${ }^{8}$ Según L. M. Capoulas, europarlamentario portugués hasta 2014 y ponente del Parlamento Europeo sobre el Reglamento de las ayudas directas de la PAC durante las negociaciones para su última reforma, dicha negociación se inició con una posición en la que el greening deberían cumplirlo todos los agricultores que declarasen más de tres hectáreas en la PAC, tope que en el acuerdo final se subió hasta 10 hectáreas. Esto significa que el $85 \%$ de los agricultores europeos no tienen que cumplir esas condiciones. Esta afirmación de L. M. Capoulas tuvo lugar en la presentación de la estrategia "Agrohorizonte 2020" de la Consejería de Agricultura y Ganadería de Castilla y León (España), en diciembre de 2014. Disponible en: $<$ http://www.agronewscastillayleon.com/el-greening-legitima-los-agricultores-como-obrerosdel-medio-ambiente>. [Consulta: 21 de diciembre de 2015]. El aumento del tope en la superficie mínima requerida para cobrar este "pago verde" y el hecho de que más de tres cuartas partes de los productores comunitarios están eximidos de su cumplimiento evidencia
} 
hectáreas de la explotación, se deben producir al menos dos tipos de cultivos distintos, sin que el principal represente más del $75 \%$ de dicha tierra de cultivo. También implica que, si la tierra de cultivo de la unidad de producción cubre más de 30 hectáreas, debe haber al menos tres cultivos diferentes, sin que el principal suponga más del $75 \%$ de la tierra de labor total, y los dos cultivos mayoritarios juntos no ocupen más del 95\% de la misma (Ministerio de Agricultura, Alimentación y Medio Ambiente, 2015 b).

Esta cuestión de la diversificación de cultivos y el mantenimiento de la tierra en barbecho supuso importantes protestas por parte de los agricultores españoles en el momento de negociar las condiciones de la última reforma de la PAC. La instauración de prácticas agropecuarias respetuosas con el ambiente y el clima deriva de unas exigencias concretas, es decir, la obligación de realizar rotaciones de los cultivos, disponer de pastos permanentes en las explotaciones y destinar un $7 \%$ de la superficie de cada finca a actividades con valor ecológico, o lo que es lo mismo, dejar tierras en barbecho. De la misma manera que la Agenda 2000 (1997) apenas tenía en cuenta a la agricultura mediterránea en sus planteamientos y propuestas, ahora, en su última modificación, los responsables agrarios de Bruselas olvidaron, consciente o inconscientemente, que, aunque estas medidas ambientales constituyan prácticas habituales en la agricultura europea, en realidad no se pueden aplicar de forma indiscriminada en cualquier lugar de Europa. Resulta evidente al respecto que no es posible dejar pastos permanentes en el aprovechamiento hortícola intensivo, practicar rotaciones de cultivos en una explotación de cítricos o dejar parcelas en barbecho en una finca consagrada al viñedo, que son las producciones más características, rentables y competitivas de la agricultura mediterránea. Ante las protestas de los países meridionales de la UE, sobre todo por parte de España y sus organizaciones agrarias, la PAC rectificó, estableciendo el pago automático de las ayudas a los agricultores que disponen de cultivos permanentes, como ya se ha indicado arriba.

En cuanto a las superficies de interés ecológico necesarias para percibir el "pago verde", cuando la explotación cuente con más de 15 hectáreas de tierra de cultivo, al menos el 5\% de dicha tierra y de las superficies que hayan sido forestadas en el marco de los programas de desarrollo rural, estará dedicada a alguna de las cuatro categorías de superficies de interés ecológico por las que, por ejemplo, ha optado España. Estas superficies, según el Ministerio de Agricultura, Alimentación y Medio Ambiente (2015 b), son las siguientes:

1) Las tierras de barbecho que no se dediquen a la producción durante al menos nueve meses desde la cosecha anterior y en el periodo comprendido entre el mes de octubre del año previo al de la solicitud y el mes del septiembre del año de la solicitud. Asimismo, las superficies de barbecho que deseen computarse como superficies de

una vez más las incongruencias de la PAC en ciertos aspectos y la relativa inutilidad de todos los requisitos exigidos en su última reforma para ser perceptor de las ayudas, burocracia que, en vez de aligerarse, se ha complicado aún más. 
interés ecológico no deberán haber estado precedidas por ningún cultivo fijador de nitrógeno.

2) Las superficies consagradas a los cultivos fijadores de nitrógeno9.

3) Las superficies forestadas en el marco de los programas de desarrollo rural.

4) Las superficies dedicadas a la agrosilvicultura que reciban o hayan recibido ayudas en el marco de los programas de desarrollo rural.

Esta norma tiene sus excepciones, pues quedan exentas de la diversificación de cultivos y del cómputo de las superficies de interés ecológico dos tipos de explotaciones:

1) Aquellas en las que más del $75 \%$ de las tierras de cultivo (tierra de labor ocupada por cultivos herbáceos, en secano o regadío, y que de modo habitual se cosechan con carácter anual, o dejada en barbecho), se utilice para producir hierba $\mathrm{u}$ otros forrajes herbáceos, o se dejen en barbecho, o se dediquen a una combinación de ambos usos, siempre que la tierra de cultivo restante $(25 \%)$ no sobrepase las 30 hectáreas.

2) Aquellas en las que más del $75 \%$ de la superficie agrícola admisible (tierras de cultivo, pastos permanentes o cultivos permanentes) sea utilizada como pasto permanente o para la producción de hierba u otros forrajes o de cultivos bajo agua durante una parte significativa del año o del ciclo de cultivo (arroz, por ejemplo), o se dediquen a una combinación de estos usos, siempre que la tierra de cultivo restante $(25 \%)$ no supere las 30 hectáreas $^{10}$.

Sin embargo, en no pocas ocasiones la realidad del día a día desmiente los buenos propósitos teóricos, ya que la organización española Cooperativas Agroalimentarias Castilla-La Mancha ${ }^{11}$ ha denunciado recientemente el problema que puede representar la reducción de las ayudas comunitarias a la producción ecológica para el bienio 2015-2016, y tras modificar la normativa que regula las ayudas a la agricultura ecológica y que afecta también a los compromisos firmados en la PAC. Este descenso alcanza el $67 \%$ de las ayudas en el caso de los cultivos herbáceos, el $41 \%$ en el olivar y el $36 \%$ en el viñedo. Por lo que respecta a los cultivos herbáceos, la reducción de las subvenciones puede conllevar la renuncia de muchos agricultores a la producción ecológica. Si los productores renuncian a este tipo de producción, podrían ver penalizadas sus ayudas por parte de la PAC, pues con las siembras ya realizadas, no cumplirían las exigencias del "pago verde" por no ser ecológicos. Si no desean que esto ocurra, deberán seguir siendo ecológicos sin cubrir los costes de cultivo, al haberse reducido sus ayudas el $67 \%$. Es decir, se trata de un dilema trágico para los agricultores, aunque también perjudicaría a la ganadería ecológica, que sólo puede

\footnotetext{
${ }^{9}$ Leguminosas grano para consumo humano o ganadero y leguminosas forrajeras.

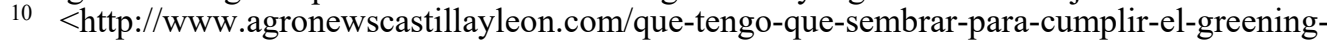
ocho-casos-practicos $>$. [Consulta: 22 de abril de 2015].

11 http://www.finanzas.com/noticias/empresas/20160311/cooperativas-avisa-gran-perjuicio3366023.html>. [Consulta: 12 de marzo de 2018].
} 
consumir cereales de origen ecológico, por lo que tendría que afrontar nuevos obstáculos para el desarrollo de su actividad.

Por lo que atañe al mantenimiento de los pastos permanentes, aprovechamiento fundamental en las zonas desfavorecidas y de montaña de España, su cumplimiento se comprobará contabilizando la superficie de pastos en todo el país y no la que ofrecen los productores de manera individual. Será el Ministerio de Agricultura, Alimentación y Medio Ambiente el encargado de calcular la proporción entre la superficie declarada dedicada a pastos permanentes en todo el país y la superficie agraria declarada total a nivel nacional. En el caso de que esta proporción disminuya en más de un $5 \%$ con respecto a la proporción del año 2015, que se tomará como proporción de referencia, se deberán tomar medidas para restaurar el nivel de referencia de pastos permanentes. Dichas medidas sí afectarán a título individual a los agricultores. Cuando haya tenido lugar la conversión de pastos permanentes a otros usos, como por ejemplo las tierras de cultivo, los agricultores causantes de este cambio tendrán la obligación de restaurar esas superficies y devolverles su utilidad como pastos permanentes. Por último, las zonas de pastos permanentes ubicadas en hábitats naturales, con flora y fauna silvestres y ambientalmente sensibles, no podrán reconvertirse a otros usos, ni labrarse, ni realizar en ellas otras labores que no sean las de su estricto mantenimiento. Es precisamente el mantenimiento de los pastos permanentes, como condición indispensable para que los agricultores tengan derecho a recibir las ayudas de la nueva política agraria, la novedad de la PAC que más controversias ha suscitado en los ganaderos de amplias áreas del territorio español.

\section{Amenazas ambientales y sociales de la Nueva PAC para el medio rural español}

El Coeficiente de Admisibilidad de Pastos (CAP) es de obligada aplicación en la UE desde el año 2007 y ajusta las superficies declaradas de pastos a las superficies reales, de acuerdo con lo previsto en los reglamentos de la PAC. Este coeficiente es el resultado del producto de los factores suelo, pendiente y vegetación, con el fin de determinar los lugares en los que puede pastar la ganadería extensiva (vacuno, ovino, caprino). Esta superficie susceptible de ser destinada al pasto del ganado se ve reducida en las ayudas comunitarias porque se descuenta el terreno considerado no apto para el aprovechamiento de los animales, es decir, el que tiene una elevada pendiente, una superficie rocosa o masas arbóreas y arbustivas.

La reforma de la PAC para el periodo 2014-2020 ha impuesto un nuevo coeficiente que excluye de las ayudas a los bosques, zonas leñosas de matorral y territorios de alta montaña en los que tradicionalmente ha pastado el ganado, sin tener en consideración que los pastores son los principales agentes de la preservación del ambiente, pues la ganadería extensiva no sólo es una fuente de riqueza, empleo y salud, sino también una actividad de vital importancia para la preservación del hábitat y la diversidad de la flora y la fauna en España. Asimismo, no es necesario insistir en la relevancia que tiene el pastoreo en la prevención y lucha contra los incendios 
forestales $^{12}$, en la mitigación del cambio climático, en la gestión territorial, en su aportación a la seguridad alimentaria y en la aportación de servicios ambientales.

Esta decisión de la nueva PAC excluye de las ayudas a cinco millones de hectáreas de pastos arbolados y a más de once millones de hectáreas de pastos arbustivos en todo el país, puesto que la UE considera que el bosque mediterráneo y otros ecosistemas protegidos no son productivos para la agricultura y la ganadería. Por citar sólo un ejemplo, el nuevo coeficiente elimina el $81 \%$ de los pastos en el pequeño municipio leonés de Villanueva de Omaña ${ }^{13}$. La ausencia de subvenciones va a dejar en la ruina a los pequeños ganaderos y pastores de las tierras más marginales, contribuyendo aún más al quebranto económico de cientos de municipios españoles y a la ya tradicional despoblación de las zonas más desfavorecidas de España ${ }^{14}$. La ganadería extensiva es, junto con el turismo, la principal fuente de ingresos y el más importante modo de vida en muchas zonas de montaña españolas, lo que ahora se ve amenazado por las restricciones de la PAC.

Con esta miopía de las autoridades de Bruselas al reclasificar muchos pastos arbolados como superficies forestales y aplicar un nuevo CAP y no considerar las especificidades de cada país ${ }^{15}$, al final se conseguirá tener un ambiente preservado pero sin vida, sin actividad, sin presencia humana, en la mejor versión del conservacionismo y de la ecología profunda ${ }^{16}$. Es precisamente el pastoreo y la ganadería extensiva los que han podido mantener la biodiversidad y los valores

\footnotetext{
${ }^{12} \mathrm{Al}$ estar los matorrales más densos y cerrados y el terreno más sucio de maleza al no pastar en ellos el ganado, el riesgo de incendio forestal aumenta de manera considerable.

$13<$ http://www.cuartopoder.es/laespumadeldia/2015/12/01/la-ue-amenaza-la-supervivenciade-los-pastores-y-la-ganaderia-extensiva-en-espana/17615>. [Consulta: 1 de diciembre de 2017].

${ }^{14}$ Un problema tradicional para la ganadería extensiva, como es la dificultad de conseguir terreno suficiente para que las explotaciones sean viables y rentables, se agrava aún más con la nueva PAC, pues la normativa vigente exige que cada parcela esté identificada con el nombre de su propietario y el régimen en el que se explota (alquiler o propiedad). Si antes ya costaba conseguir cesiones, alquileres o ventas de terrenos para pastos, ahora será todo más complicado al exigir que todo se haga de manera oficial y por escrito. $<$ http://www.lavozdegalicia.es/noticia/ourense/2015/12/11/productores-razas-autóctonas-venfuturo-comercializacion-directa/0003_201512011C7991.htm $>$. [Consulta: 11 de diciembre de 2017].

${ }^{15}$ La PAC tendría que asumir que no es lo mismo un prado holandés, alemán o francés que las zonas de pastos de montaña o las dehesas en España. Estas últimas son bosques claros de encinas y alcornoques, a veces con otras especies, con pastizal y matorral, donde se llevan a cabo de manera integrada actividades ganaderas, agrícolas, forestales y cinegéticas.

${ }^{16}$ En 1973, el montañista noruego A. Naess introdujo el término movimiento de ecología profunda en la literatura ecológica. Se trata de un tipo de ecología que no considera la existencia humana y aspira a conservar el medio, pero sin la presencia de los seres humanos. La ideología que subyace en este modelo se basa en la visión de las personas como entes necesariamente destructores de la naturaleza.
} 
naturales y ambientales en las zonas más inhóspitas del país, donde han aprovechado sus recursos vegetales (pastos leñosos y de montaña) de manera tradicional ${ }^{17}$.

Por otro lado, muchos ganaderos verán reducida la superficie declarada de pastos aprovechados por sus ganados, lo que implicará una reducción de las ayudas percibidas hasta finales de 2020, año hasta el que se prolongará el cómputo de las hectáreas solicitadas para pastos y que se calcularon en 2015. Para evitar esta situación, no ha faltado quien ha adquirido más animales y aumentado la carga ganadera (Unidades Ganaderas/Hectárea), actitud que perjudica al ecosistema y comporta la pérdida total tanto de las indemnizaciones compensatorias en las zonas desfavorecidas y de montaña como de las ayudas agroambientales por parte de la $\mathrm{PAC}^{18}$.

El declive del pastoreo, empujado por las políticas restrictivas de la UE y la falta de apoyo de las autoridades españolas, pone en peligro el potencial económico, social, cultural, alimentario y ambiental de la ganadería extensiva en España. La PAC discrimina a la ganadería extensiva por lo ya comentado y por unas exigencias sanitarias casi imposibles de cumplir, y que parecen pensadas sólo para las grandes concentraciones de animales estabulados, es decir, para la ganadería intensiva o industrializada. Incluso las políticas de conservación de la naturaleza, que deberían defender esta actividad como garante de los paisajes culturales y de unos hábitats valiosos, en realidad contribuyen a que los pastores abandonen la actividad y en ocasiones el propio medio rural, perdiendo así un patrimonio de gran trascendencia histórica y cultural ${ }^{19}$. Incluso se puede perder para siempre la riqueza genética y biológica que representan las razas autóctonas, algunas de ellas en peligro de extinción.

Aparte de las nuevas normas de la PAC, que fieles a su tradición no han desarrollado nunca una política específica de la UE para las zonas de montaña, el gobierno español tampoco ha hecho mucho por salvaguardar la ganadería extensiva en las zonas más desfavorecidas del país, evitar el abandono de las áreas rurales más abruptas y compensar con dotaciones presupuestarias las dificultades que conlleva vivir y trabajar en estos lugares. Incluso no falta quien sostiene que el Ministerio de Agricultura, Alimentación y Medio Ambiente español no ha sabido defender el monte

${ }^{17} \mathrm{La}$ amenaza que la nueva PAC representa para el ambiente, la conservación de la naturaleza y la población rural en España, así como el valor del pastoreo extensivo y de la trashumancia en la preservación de la biodiversidad, la fertilidad del suelo y el control de los incendios forestales, son aspectos que se expusieron en un encuentro celebrado en Madrid en diciembre de 2015, con la organización de la Asociación Trashumancia y Naturaleza. $<$ http://www.gaceta.es/noticias/ecologistas-ganaderos-analizaran-amenazas-ambientalesnueva-pac-09122015-1424>. [Consulta: 9 de diciembre de 2017].

$18<\mathrm{http}$ ://www.campogalego.com/es/desarrollo-rural/denuncian-la-supresion-de-las-ayudas-dela-pac-para-los-pastos-arbustivos-y-bajo-arbolado-de-menos-de-03-hectareas $>$. [Consulta: 11 de diciembre de 2017].

$<$ http://www.ecoticias.com/naturaleza/109398/Defensa-ganaderia-extensiva $>$. [Consulta: 25 de noviembre de 2017]. 
mediterráneo como hecho diferencial respecto a los bosques del norte de Europa ${ }^{20}$. A este respecto habría que tener en cuenta que es perfectamente posible llegar allí donde no alcanzan las subvenciones europeas. Así lo demuestran la Junta de Andalucía, que ha reconocido y protegido el valor y la especificidad de su dehesa frente a la normativa comunitaria, y la Consejería de Agricultura y Ganadería de Castilla y León, que revisó todos los expedientes afectados por la reducción del CAP para evitar en la medida de lo posible que los ganaderos salieran gravemente perjudicados por la reducción indiscriminada de dicho coeficiente ${ }^{21}$.

\section{Conclusiones}

Las sociedades se encuentran cada vez más concienciadas respecto a la sobreexplotación y agotamiento de los recursos y a la pérdida de su patrimonio natural. De ahí que la pretensión de extender las exigencias ambientales que deben cumplir los agricultores para poder percibir una parte importante de las ayudas europeas (un 30\%) parezca más destinada a congraciarse con la sociedad urbana (mayoritaria por otro lado en el mundo desarrollado) y con los grupos ecologistas que al logro de unos resultados fehacientes en el medio natural. Es loable el intento de evitar la contaminación, cuidar el ambiente y preservar los recursos naturales, nadie sensato podría oponerse a ello, pero los agricultores y ganaderos temen que estas medidas resulten difíciles de cumplir, causen mermas en las cosechas y eleven los gastos de producción. Y eso por no hablar del apoyo que reciben la agroindustria y las grandes explotaciones de monocultivo, que precisamente contaminan más que las pequeñas unidades de producción agropecuaria.

Estas iniciativas europeas de tipo ambiental entran en flagrante contradicción con la presión que ejercen las compañías transnacionales de la agroalimentación en las autoridades comunitarias en busca de salvaguardar sus intereses y el férreo control que sobre los mercados tiene la gran distribución agroalimentaria organizada (Segrelles, 2010). Tanto estas firmas como los gobiernos conniventes son los responsables de que los agricultores de los países subdesarrollados trabajen en el campo durante doce o más horas al día, sin seguridad social ni derechos de ningún tipo, y de que muchos de estos países no estén obligados a cumplir la más elemental norma en la producción de alimentos, en la seguridad de los mismos o en la protección ambiental. No es fácil, por lo tanto, comprender la razón del nivel de exigencia a los que se somete a los agricultores europeos en estas materias, sobre todo

\footnotetext{
${ }^{20}<$ http://www.laopiniondezamora.es/agroalimentacion/2016/01/02/cambios-pac-perdidarenta-principales/895126.html>. [Consulta: 4 de enero de 2017].

${ }^{21}<\mathrm{http}$ ://www.campogalego.com/es/desarrollo-rural/denuncian-la-supresion-de-las-ayudas-dela-pac-para-los-pastos-arbustivos-y-bajo-arbolado-de-menos-de-03-hectareas $>$. [Consulta: 11 de diciembre de 2017].
} 
por lo que respecta a las cuestiones ambientales. En este contexto, en el que las empresas transnacionales europeas prefieren comprar materias primas y alimentos baratos en el exterior antes que producir caro en la propia UE, es evidente que por muy bien que hagan las cosas los agricultores comunitarios, y especialmente los españoles, resulta muy difícil mantener el modelo de calidad y seguridad alimentaria por el que tanto se ha luchado en la $\mathrm{UE}^{22}$.

El actual discurso ecológico y rural de la UE oculta en realidad un movimiento claro hacia la liberalización de los mercados agrícolas europeos y la selección y especialización de las explotaciones más competitivas. Los nuevos discursos ambientales y rurales producen en la UE un auténtico desorden territorial de la producción, ya que la concentran en las regiones más rentables y causan la despoblación de las zonas más desfavorecidas. En este proceso se excluye a las unidades de producción familiares, que son las que precisamente conceden contenido social y demográfico al campo y mantienen una agricultura rentable y en funcionamiento y un medio rural vivo. Entre los años 2005 y 2013 desparecieron 3,7 millones de explotaciones en la UE, lo que supone un descenso del $26 \%$, pues se pasa de 14,4 millones a 10,7 millones de unidades de producción. La pérdida proporcional de las explotaciones ganaderas ha sido aún mayor: el $32 \%$. Se ha pasado de 9 a 6,1 millones de explotaciones en el mismo periodo de tiempo. Sin embargo, el tamaño de estas explotaciones sigue la tendencia opuesta. Las granjas de porcino, vacuno de leche y aves de corral son cada vez mayores, más concentradas y más especializadas (Greenpeace, 2019: 6).

Aunque son fundamentales las medidas para la protección del ambiente y el clima en las actividades agropecuarias, el verdadero problema de fondo en el campo español y europeo radica en otros factores que deberían modificarse si se desea que la agricultura siga siendo una actividad vinculada al territorio y también primordial para mantener a la población en el medio rural y salvaguardar los ecosistemas. P. López Bermejo y M. Á. de Porras (2016) afirman que durante los últimos años han surgido diversas opciones productivas que pueden convertirse en el embrión del cambio necesario en la agricultura. Mencionan, por ejemplo, la agricultura ecológica, modelo del que puede partir una producción más sostenible desde el punto de vista ambiental y que sea generadora de empleo. También hacen alusión al nacimiento de redes

\footnotetext{
${ }^{22}$ A este respecto puede consultarse la opinión de Óscar Herrero de Frutos en el siguiente enlace: <http://www.acueducto2.com/opinion-con-las-cosas-de-comer-no-se-juega/32208>. [Consulta: 20 de marzo de 2016]. A finales del mes de abril de 2016 se reiniciaron las negociaciones sobre el tratado de libre comercio entre la UE y el Mercosur, conversaciones que llevaban muchos años paralizadas. Francia, con el apoyo de otros doce países miembros, intentó bloquear este pacto comercial para no perjudicar a la agricultura europea ni soliviantar a sus agricultores. Se argumentan diversas cuestiones ambientales y sociales, pero el principal obstáculo estriba en que los socios del Mercosur (Argentina, Brasil, Paraguay y Uruguay) son más competitivos en muchas "producciones sensibles" para la UE (Diario El País, Madrid, 26 de abril de 2016: 5). En cualquier caso, lo que es bueno para las compañías transnacionales agroalimentarias europeas, puede resultar nefasto para el campo comunitario.
} 
alternativas de consumo, que reducen el coste energético de la distribución y acortan la distancia entre el productor y el consumidor, así como a ciertas experiencias que batallan por el acceso a la tierra, la permanencia de las pequeñas y medianas explotaciones, la creación de bancos de tierras públicas o la formación de cooperativas. Sin embargo, los mismos autores no dudan en aseverar que todo esto nunca será suficiente sin un proyecto político que sostenga y profundice estas iniciativas, afirmación que debería constituir una llamada de atención para todos aquellos que, con una visión fatalista, consideran que las políticas agrarias europeas se encuentran maniatadas por las exigencias de la OMC en cuanto a los precios y el libre mercado, que es necesario que las pequeñas y medianas explotaciones desaparezcan y que sólo perduren las que consigan ser competitivas o que el retroceso de la agricultura es un signo inequívoco de desarrollo y modernización.

La PAC podría representar este papel político por su capacidad y por ser la única política verdaderamente común a todos los países de la UE. Sin embargo, su orientación hacia un mercado sin controles ni regulación y la necesidad de seguir siendo un actor principal en el comercio agroalimentario mundial, hacen que no haya sido capaz, tampoco en su última reforma, de mantener los niveles de renta en la agricultura, frenar la despoblación rural, garantizar el relevo generacional en el campo, mejorar el reparto de la revalorización que supone la transformación y distribución de los productos y asegurar que sus ayudas no vayan a los grandes latifundistas y agroindustrias, sino a quiénes más las necesitan, es decir, los pequeños y medianos agricultores que viven en el campo y del campo. En cualquier caso, la sostenibilidad y las exigencias ambientales no tienen por qué excluir el apoyo a la modernización, el reparto de rentas más justo y la equidad social en el campo europeo. Ante esto, los principios de mercado, prevalentes en la actualidad, actúan como un auténtico freno para el desarrollo integral de las actividades agropecuarias y forestales y los espacios rurales de la UE.

Siguiendo la lógica capitalista y las tendencias de las sociedades postindustriales, las explotaciones menos competitivas deberían desaparecer por ineficientes e inútiles para el sistema. Sin embargo, no se puede acabar con la agricultura familiar de repente y de forma agresiva y contundente porque esto daría lugar a grandes protestas en la mayoría de los países comunitarios, sobre todo en los que tienen una importante base agropecuaria, como es el caso de España o Francia. La estrategia consiste en hacerlo de manera sutil, poco a poco, casi de forma imperceptible, otorgando subvenciones y protegiendo la agricultura y, por otro lado, dejando que actúe el mercado, que es el mecanismo que pondrá a cada cual en su sitio de modo inapelable. La prueba está en el intenso proceso de concentración de la producción agropecuaria que ha tenido lugar en los países de la UE durante las últimas décadas y la consiguiente desaparición de millones de pequeñas y medianas explotaciones de tipo familiar, como ya se ha comentado más arriba.

La producción de carne de cerdo en las granjas más grandes (con una producción valorada en medio millón de euros o más) tenía en España una concentración del 45\% en 2004 y del $64 \%$ en 2016. En el mismo periodo de tiempo, estos porcentajes eran 
del $29 \%$ y del $70 \%$ en Bélgica, del $61 \%$ y del $94 \%$ en Dinamarca o del $47 \%$ y del 82\% en los Países Bajos (Greenpeace, 2019: 10).

Esta situación de asfixia económica y de aguda descapitalización que tiene lugar en las explotaciones agrarias familiares es lo que la PAC debería solucionar de manera radical y no prolongar la agonía con subvenciones que reducen al agricultor profesional a la mera condición de perceptor de ayudas públicas, necesarias por otro lado, pero que apenas contribuyen a soslayar las crisis recurrentes, los vaivenes bruscos de los mercados o la crónica falta de rentabilidad y competitividad en el campo. Resulta cínico e hipócrita conceder subvenciones que desmotivan más que otra cosa, aun reconociendo su necesidad ${ }^{23}$, y por otro lado tener la vista puesta ante todo en los mercados exteriores, en la prestación de ayudas multimillonarias a los grandes terratenientes ${ }^{24}$, absentistas, especuladores y empresas de diverso signo, en congraciarse con el poder omnímodo de la gran distribución organizada agroalimentaria y en consentir la existencia de unos precios en origen indignos para los productores familiares.

23 Justo es reconocer que sin los mecanismos de ayuda desarrollados por la PAC la desaparición de las explotaciones pequeñas y más vulnerables habría sido más intensa y un número mucho mayor de áreas rurales estaría despoblado en la actualidad. Muchísimas explotaciones de amplias áreas españolas tienen seriamente condicionada su supervivencia a corto plazo. Si muchas de ellas continúan en activo es gracias a las ayudas públicas que reciben de la UE. De no ser por las subvenciones de la PAC, estas unidades de producción no alcanzarían siguiera el umbral de rendimiento efectivo, es decir, aquel en el que no se tiene en cuenta el valor de la mano de obra familiar en la explotación. Esto es lo que ocurre con muchas pequeñas explotaciones de ganado vacuno de leche en varias zonas de Galicia. Disponible en: <http://www.lavozdegalicia.es/noticia/barbanza/2016/04/26/ocho-diezexplotaciones-lacteas-sobreviven-gracias-ayudas-publicas/00031461614847988545559.htm>. [Consulta: 26 de abril de 2016]. Sin embargo, reconocer el papel de la PAC en el mantenimiento de la actividad agropecuaria en muchas zonas no significa que haya que ignorar sus desequilibrios, injusticias, incongruencias, prioridades y equivocaciones. Merece la pena destacar al respecto la opinión de un sector cada vez más importante del gobierno socialista francés cuando dice que la PAC y sus recursos han hecho poco por la creación de empleo en el medio rural y que tan sólo han servido para mantener los 22 millones de puestos de trabajo de la agricultura en Europa. Disponible en: $<$ http://www.elboletin.com/internacional/133662/socialistas-franceses-pie-guerra-pac.html>. [Consulta: 1 de mayo de 2017].

${ }^{24}$ En Francia, el colectivo Inventons Demain, en el entorno del partido socialista, ha calificado a la PAC como la "ley del más fuerte" y caracterizada por el "darwinismo". Disponible en: $<$ http://www.elboletin.com/internacional/133662/socialistas-franceses-pie-guerra-pac.html>. [Consulta: 1 de mayo de 2017]. 


\section{Bibliografía}

Bardají, I. (coord.) (2014): Reflexiones en torno a la PAC. Serie Economía, 22, Almería, Cajamar Caja Rural.

Comisión Europea (2014): Comprender las políticas de la Unión Europea: Agricultura. Una asociación entre Europa y los agricultores. Política Agrícola Común de la UE: por nuestra alimentación, nuestro campo y nuestro medio ambiente. Luxemburgo, Oficina de Publicaciones de la Unión Europea.

Delgado Serrano, M. M. (2004): La política rural europea en la encrucijada. Madrid, Ministerio de Agricultura, Pesca y Alimentación.

Fundación de Estudios Rurales (2013): Agricultura familiar en España. Anuario 2013. Madrid, Fundación de Estudios Rurales-Unión de Pequeños Agricultores.

Klein, N. (2015): Esto lo cambia todo. El capitalismo contra el clima. Barcelona, Paidós.

Roca Torrent, A. y Ricart Casadevall, S. (2014): La PAC y los bienes comunes, un ejercicio teórico-práctico como estrategia de futuro. XVIII Coloquio de Geografía Rural. Girona, septiembre.

Rutz, C., J. Dwyer and J. Schramek, Jorg (2013): More New Wine in the Same Old Bottles? The Evolving Nature of the CAP Reform Debate in Europe, and Prospects for the Future. Sociologia Ruralis, 54, 3, 266-284.

Segrelles Serrano, J. A. (2010): La distribución agroalimentaria y su influencia en la pobreza campesina. Scripta Nova. Revista Electrónica de Geografía y Ciencias Sociales, XIV, 325, 1-26. Disponible en: <http://www.ub.es/geocrit/sn/sn-325.htm>. [Consulta: 21 de junio de 2015].

Segrelles Serrano, J. A. (2017): Las ayudas agrarias y sus repercusiones sobre la agricultura familiar en la última reforma de la Política Agraria Común (2014-2020) de la Unión Europea: ¿Cambiar todo para que todo siga igual? Boletín de la Asociación de Geógrafos Españoles, 74, 161-183.

Westhoek, H. et al. (2014): Food Choices, Health and Environment: Effects of Cutting Europe's Meat and Dairy Intake. Global Environmental Change, 26, 196-205.

\section{Recursos electrónicos}

Capoulas Santos, L. M. (2014): Agrohorizonte 2020, Consejería de Agricultura y Ganadería de Castilla y León, Segovia. Disponible en: < http://www.agronewscastillayleon.com/elgreening-legitima-los-agricultores-como-obreros-del-medio-ambiente $>$. [Consulta: $21 \mathrm{de}$ diciembre de 2015].

Comisión Europea (1997): Agenda 2000: por una Unión más fuerte y más amplia, Bruselas.

Disponible

en:

$<$ http://repositori.uji.es/xmlui/bitstream/handle/10234/55829/Suplemento\%205-

97.pdf? sequence $=1>$. [Consulta: 29 de agosto de 2017]. 
Fanjul, G. (2002): La necesidad de ser coherentes. Reforma de la PAC y países en desarrollo. Oxfam International. Disponible en:

$<$ http://fongdcam.org/manuales/comerciojusto/datos/docs/ARTICULOS\%20Y\%20DOCUME NTOS\%20DE\%20REFERENCIA \%20def/CONCEPTO $\% 20 \mathrm{CI} / \mathrm{Como} \% 20$ funciona $\% 20 \mathrm{el}$ \%20sistema/12-coherencia\%20PAC.pdf>. [Consulta: 27 de agosto de 2017].

Ferrer, J. (2005): PAC, ¿reforma o liquidación? Revista El Ecologista, 46, diciembre, 1-6. Disponible en: <http://www.ecologistasenaccion.es/article8133.html $>$. [Consulta: $27 \mathrm{de}$ agosto de 2016].

Fischer-Boel, M. (2008): Hacia una nueva PAC, Espacio Europa. Disponible en: $<$ http://blog.espacioeuropa.eu/análisis/la-solidaridad-europea-en-cataluna $>$. [Consulta: 22 de mayo de 2008].

Greenpeace (2019): Alimentando el problema. La peligrosa intensificación de la ganadería europea. Disponible en: >https://es.greenpeace.org/es/wocontent/uploads/sites/3/2019/02/20190209_AlimentandoElProblema_PAC.pdf $>$. [Consulta 19 de febrero de 2019].

Herrero de Frutos, Ó. (2016): Con las cosas de comer no se juega. Acueducto2.com, 19 de marzo. Disponible en: <http://www.acueducto2.com/opinion-con-las-cosas-de-comer-nose-juega/32208>. [Consulta: 20 de marzo de 2016].

López Bermejo, P. y Porras, M. Á. (2016). La agricultura en un nuevo modelo productivo. Diario Público, 3 de marzo. Disponible en: <http://blogs.publico.es/otrasmiradas/6054/laagricultura-en-un-nuevo-modelo-productivo $>$. [Consulta: 3 de marzo de 2016].

Ministerio de Agricultura, Alimentación y Medio Ambiente (2015a): Política Agrícola Común 2015-2020. Condicionalidad. Nota Técnica $\mathrm{N}^{\mathrm{o}}$ 07, Madrid. Disponible en: $<$ http://www.magrama.gob.es/es/prensa/F7\%20Condicionalidad\%20red_tcm7362105_noticia.pdf $>$. [Disponible en: 18 de abril de 2018].

Ministerio de Agricultura, Alimentación y Medio Ambiente (2015b): Política Agrícola Común 2015-2020. Pago para prácticas beneficiosas para el clima y el medio ambiente. Nota Técnica $\quad \mathrm{N}^{\mathrm{O}} \quad 03, \quad$ Madrid. $\quad$ Disponible $<$ http://www.magrama.gob.es/es/prensa/F3\%20Pr\%C3\%A1cticas\%20beneficiosas\%20par $\mathrm{a} \% 20 \mathrm{el} \% 20 \mathrm{clima} \% 20 \mathrm{y} \% 20 \mathrm{el} \% 20$ medio $\% 20$ ambiente $\% 20 \% 20$ red_tcm $7-362101$ noticiapdf>. [Consulta: 18 de abril de 2018]. 